\title{
Minireview
}

\section{RNA editing in metazoan mitochondria: staying fit without sex}

\author{
G. Valentin Börner ${ }^{1}$, Shin-ichi Yokobori², Mario Mörl, Marion Dörner, Svante Pääbo* \\ Institute of Zoology, University of Munich, PO Box 202136, D-80021 Munich, Germany
}

Received 18 March 1997

\begin{abstract}
RNA editing subsumes a number of functionally different mechanisms which have in common that they change the nucleotide sequence of RNA transcripts such that they become different from what would conventionally be predicted from their gene sequences. RNA editing has now been found in the organelles of numerous organisms as well as in a few nuclear transcripts. Most recently, it was shown to affect tRNAs in the mitochondria of several animals. The occurrence and evolutionary persistence of RNA editing is perplexing since backmutations in the genes might be assumed rapidly to eliminate the need for 'correction' of the gene sequences at the posttranscriptional level. Here, we review the recent RNA editing systems discovered in animal mitochondria and propose that they have arisen as a mechanism counteracting the accumulation of mutations that occurs in asexual genetic system.
\end{abstract}

(C) 1997 Federation of European Biochemical Societies.

Key words: RNA editing; Mitochondria; Evolution; Müller's ratchet; tRNA

\section{Asexuality of animal mitochondria}

During every generation, the nuclear genomes of animals become reassembled anew by meiotic recombination and subsequently merge with genomes from other individuals. By contrast, the mitochondrial genomes of animals lead lonesome, asexual existences. Their inability to engage in sex has two reasons. First, in most cases, mitochondrial (mt)DNA is inherited exclusively from mother to offspring [1]. For example, in humans, in $10000 \mathrm{mtDNAs}$ no paternal molecule can be detected (C. Kilger, unpublished). The maternal pattern of inheritance is further underscored by the fact that in mice, where paternal contribution to the mtDNA of the offspring has been reported [2], this seems to be restricted to crosses between different species whereas in conspecific crosses, the paternal mtDNA becomes eliminated [3].

Second, even if mitochondria with two types of mtDNA are present in the same cell, recombination between them seems to be extremely rare. For example, in the blue mussel, where paternal mtDNA is passed down the male line and encounters maternal mtDNA from the female line in every male individual $[4,5]$, the high degree of DNA sequence divergence between the two gender-associated lineages of mtDNA [6] sug-

\footnotetext{
${ }^{*}$ Corresponding author.

${ }^{1}$ Present address: Department of Molecular and Cellular Biology, Harvard University, 7 Divinity Av., Cambridge, MA, USA.

${ }^{2}$ Present address: Department of Molecular Biology, Tokyo University of Pharmacy and Life Sciences, 1432-1 Horinouchi, Hachioji, Tokyo 192-03, Japan.
}

gests that they do not recombine [7]. Since at least one enzymatic activity promoting homologous recombination exists in mammalian mitochondria [8] this lack of genetic recombination may be due to the physical separation of the mtDNAs in different organelles. This is supported by the observation that when two non-allelic mutations are introduced within distinct organelles into human cells, no complementation is observed [9]. Thus, due to a predominance of maternal inheritance and separation of mtDNA into different organelles, mitochondrial genomes do not, or only extremely rarely, indulge in recombination. Interestingly, the fact that the mitochondrial genome seems to thrive in the absence of genetic recombination poses a dilemma.

\section{A sexual dilemma}

Müller [10] first pointed out that genomes lacking recombination and segregation will accumulate mildly deleterious mutations resulting in a gradual loss of fitness of the entire population (Fig. 1). This is an essentially irreversible process, since random genetic drift may cause a fraction of the mutated genomes to come to fixation in the population which is especially likely if the population is small. Assuming similar mutation rates for all sites, backmutations at a given site are less likely than additional mutations at any other sites. Therefore, mutations are unlikely to be reversed $[7,10]$. Because of its irreversible nature, this phenomenon has been dubbed Müller's ratchet [11]. It is relieved in the nuclear genome of sexually reproducing organisms by recombination, which allows unmutated genomes to be reconstituted by combining non-mutated parts from different genomes (Fig. 1).

Since animal mitochondria lack genetic recombination, and since mtDNA molecules go through a bottleneck of less than $200 \mathrm{mtDNA}$ during oogenesis [12], Müller's ratchet most certainly applies to the population of genomes in animal mitochondria $[13,34]$. Yet, ever since the last common ancestor of

Fig. 1. Schematic illustration of Müller's ratchet. Above, the situation in mitochondria is shown. In a mitochondrial lineage, a slightly deleterious mutation ( $)$ occurs in one molecule. If this mutation comes to fixation in the germ line, a further slightly deleterious mutation will eventually occur, that in turn will have a certain chance to come to fixation in the germ line. Thus, the numbers of deleterious mutations per genome will increase with time. Below, the situation in the nuclear genome is shown. Here, two individuals are shown, each carrying two homologous chromosomes. When deleterious mutations occur ( $\bullet$ ), they can become eliminated from one chromosome by recombination. In addition, segregation allows for unmutated chromosomes to come together in some diploid individuals, whereas other individuals carrying multiple mutations may become eliminated by selection. Thus, the numbers of deleterious mutations per genome do not increase over time. 


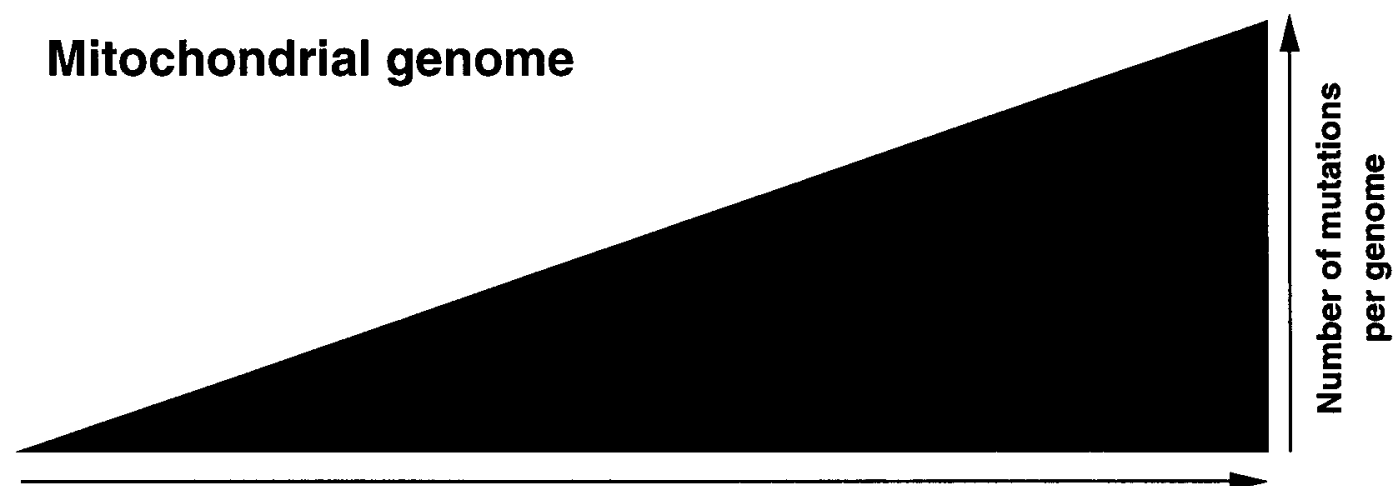

Number of generations

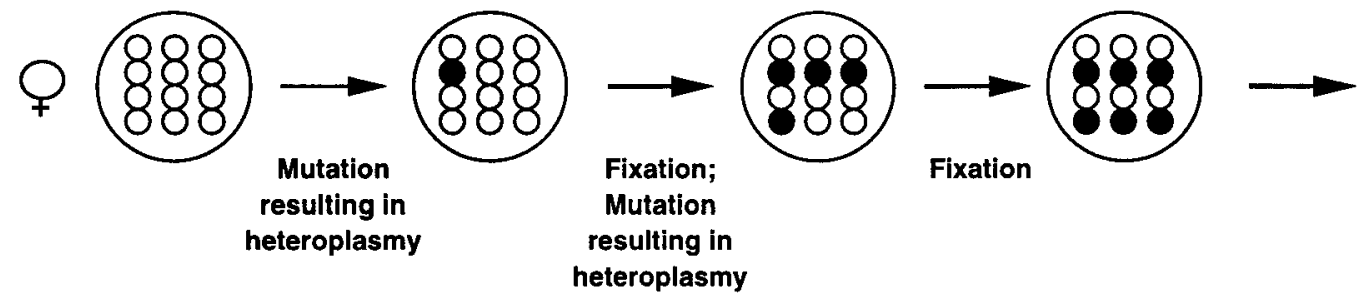

\section{Nuclear genome}
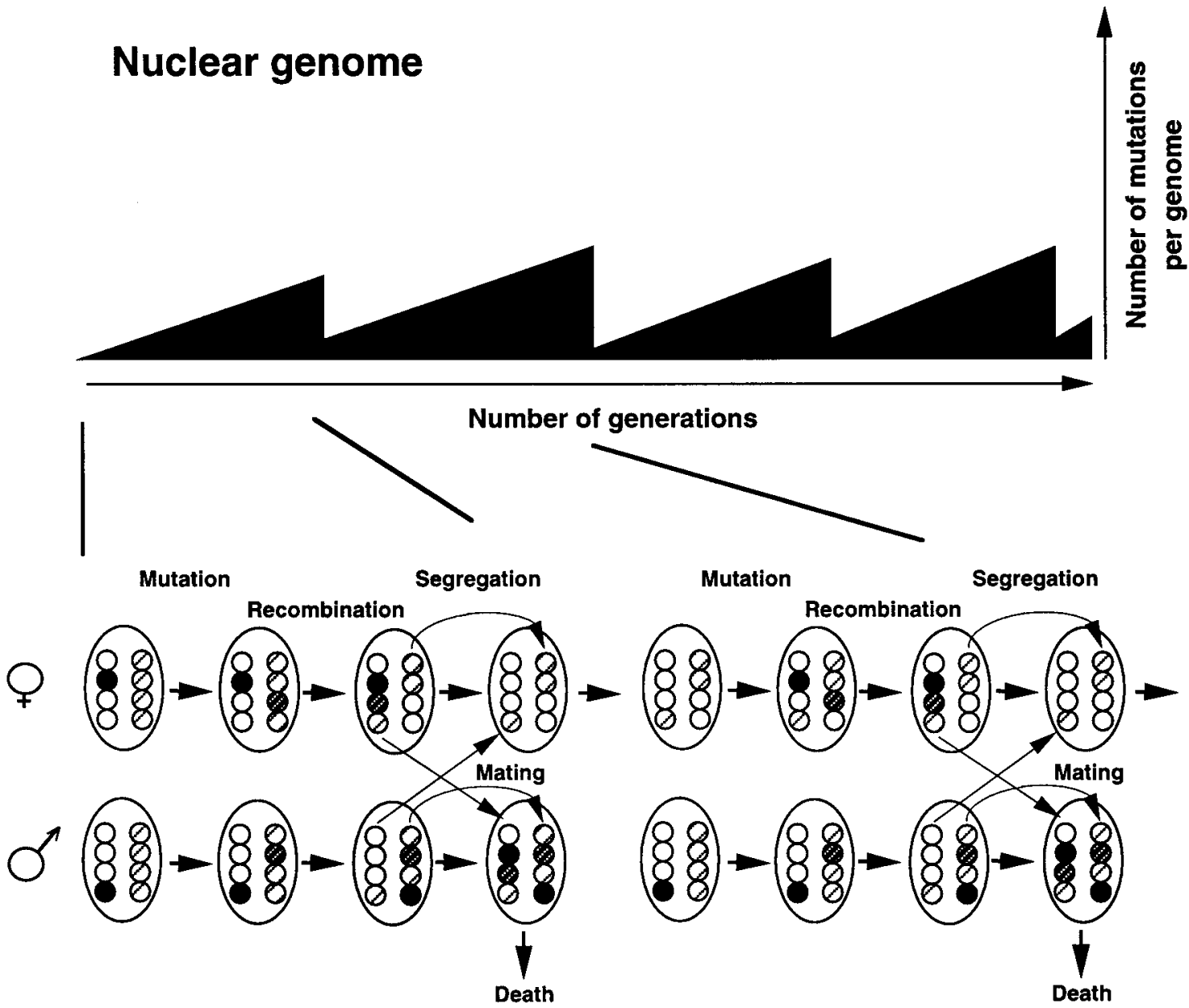
animals hundreds of millions of years ago, almost all metazoan mitochondria have preserved the same set of 13 proteincoding genes, 22 tRNA and 2 rRNA genes [14] and have continued to perform respiration sufficient to keep animals alive and breathing. Thus, against all expectations, mitochondria seem to thrive in celibacy.

However, recent studies have revealed cracks in this 'gutbürgerliche' facade. Most of these subtle signs of decay come from studies of mitochondrial tRNA genes. For example, it has been demonstrated that the average rate of nucleotide substitution in mitochondrial tRNA genes is elevated by a factor of 5 (for invertebrates) and 25 (for mammals) relative to the equivalent genes in the nucleus [13]. This is accompanied by an increased incidence of A-T and non-Watson/Crick base pairs in tRNA stem structures, an increased variation and average reduction in loop sizes, and an absence of nucleotide conservation in sites involved in tertiary structure interactions $[13,15]$. In fact, the $\mathrm{T}$ - and/or the $\mathrm{D}$-arms are replaced by smaller loop structures in nematode tRNAs [14], in the tRNA $^{\text {Ser }}$ (AGY) of all animals, the tRNA ${ }^{\text {Lys }}$ of marsupials [16] and the tRNA ${ }^{\mathrm{Cys}}$ of some reptiles [17]. It has even been suggested that the reduced rates of in vitro aminoacylation of mitochondrial tRNAs relative to procaryotic tRNAs $[18,19]$ may reflect a reduced functional efficiency of the former tRNAs [13]. Thus, there are indications that the gene products of mitochondria may after all suffer under the workings of Müller's ratchet.

\section{RNA editing of tRNAs transcribed from overlapping genes}

Another unusual feature of mitochondrial tRNA genes is that some genes overlap by one to six nucleotides with other genes that are located on the same strand and which often (but not always) encode other tRNAs (e.g. [20,21]). It has been suggested that these tRNAs might be processed such that alternatively one or the other of the two tRNAs would be cleaved out in full length from the polycistronic transcript while the other would become truncated and degraded [22]. However, in many cases, the gene sequences of the tRNAs encoded upstream suggest that several mismatches would occur in the acceptor stems of these tRNAs. Sequence analysis of cDNAs from several tRNAs transcribed from overlapping mitochondrial genes in land snails revealed that such mismatches are removed by the replacement of nucleotides in the $3^{\prime}$-parts of the acceptor stems [23]. Subsequently, this type of RNA editing has been shown to occur also in mammals [24], squid [25] and birds [26]. The replacements observed are substitutions from $G, C$, and $U$ to $A$ as well as, in the monotreme mammal platypus from $\mathrm{A}$ and $\mathrm{U}$ to $\mathrm{C}$ or $\mathrm{A}[23-$ 26].

In principle, two pathways for the editing reaction can be envisioned. The reaction could either start from the full-length upstream tRNA created by alternative processing, followed by the replacement of the mismatched bases in the acceptor stem. Alternatively, the upstream tRNA could be cleaved out from the primary transcript in a truncated form (upstream of the first mismatch) and be completed by a 'fill in' reaction. The fact that one quite readily detects upstream tRNA molecules that are truncated in their 3 '-ends [26] but not downstream tRNAs with truncated 5 '-ends [25] supports the 'fill-in' model. Furthermore, an in vitro system that processes the polycistronic primary transcript such that a truncated upstream

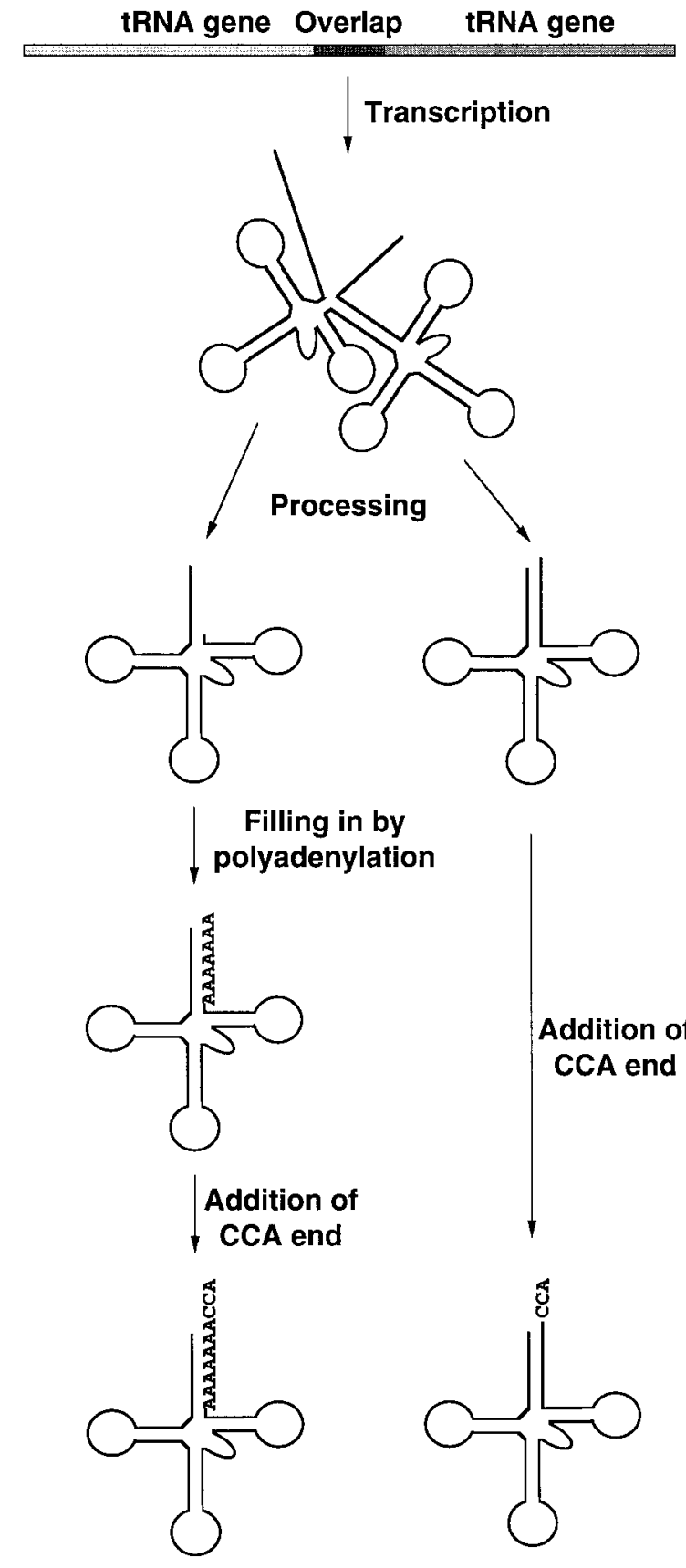

Fig. 2. A possible mechanism for the editing of the $3^{\prime}$-ends of tRNAs transcribed from overlapping genes. The primary transcript is processed such that the downstream tRNA emerges intact while the upstream one lacks bases at its $3^{\prime}$-end. These are filled in by a reaction that at least in some cases is related to polyadenylation. Subsequently, CCA-ends are added to both transcripts.

and a full-length downstream tRNA is formed has recently been established (M.M. unpublished observation). Thus, the tRNA located downstream will presumably be cleaved out in an intact form while the $3^{\prime}$-end of the upstream tRNA is completed by an RNA editing reaction (Fig. 2). It is noteworthy that this type of RNA editing seems possible only when an insertion of an A (or possibly a C) at the discriminator will result in a functional tRNA since only such tRNA genes have been found to overlap with downstream genes in metazoans [26]. The enzymatic activity responsible for the 


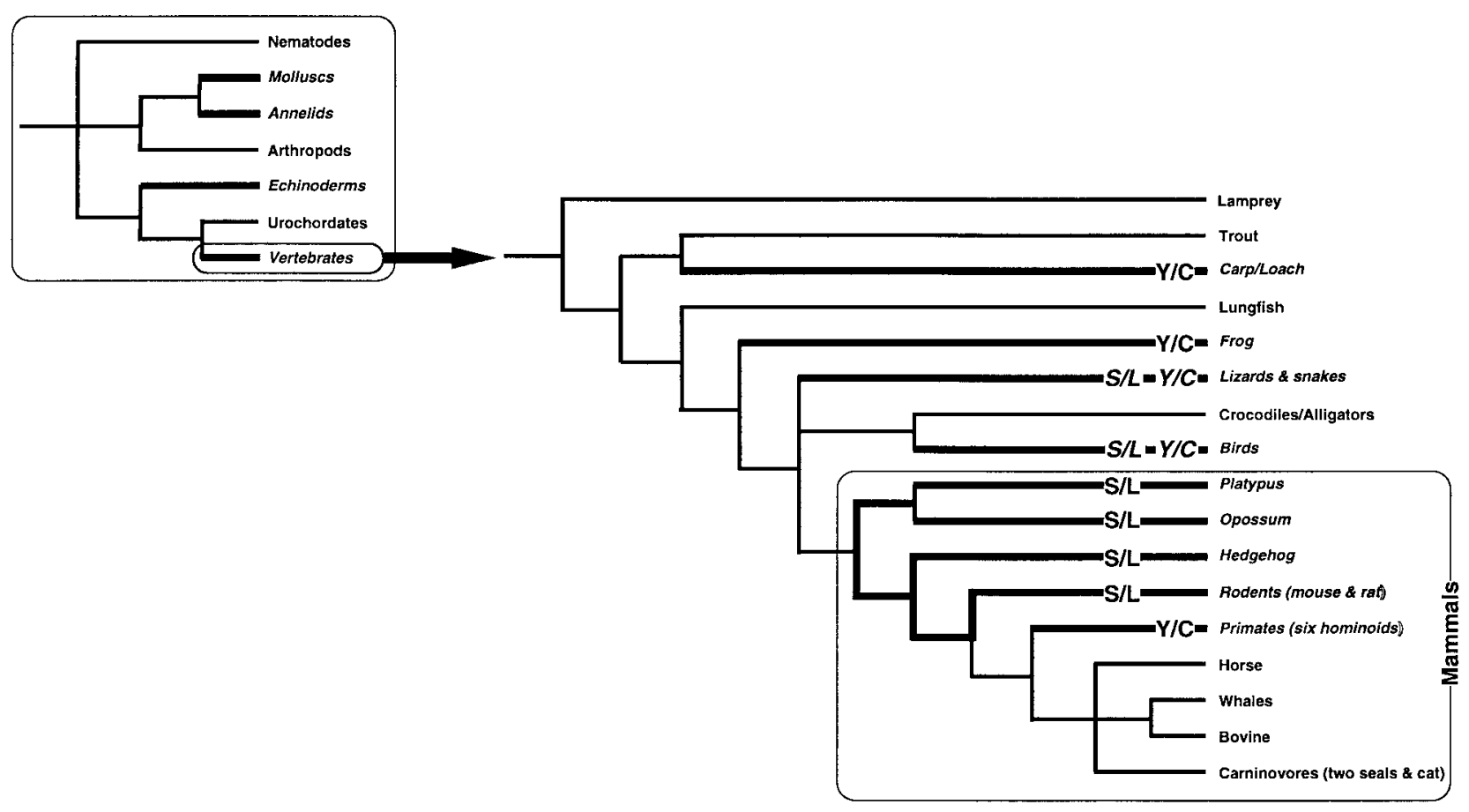

Fig. 3. Evolutionary tree showing how overlaps of tRNA genes have emerged and disappeared several times during evolution. In molluscs, annelids and echinoderms, many overlaps (thick lines and italicized names) occur. In vertebrates, only the tyrosyl-(Y) and cysteyl-(C), and seryl(S) and leucyl-(L) tRNA genes, respectively, overlap. These Y/C overlaps have either evolved independently five times (on the line to carp/ loach, frog, lizards/snakes, birds and primates), or it may have evolved once and been independently lost many times (or combinations thereof). The S/L overlap may have evolved once in the ancestor of non-amphibian tetrapodes and been lost on the line to crocodiles and alligators and once among placental mammals.

editing may be related to a poly-A polymerase since presumptive intermediates with an oligo-A tail added to the $\mathrm{RNA}^{\mathrm{Tyr}}$ can be detected in chicken [26]. However, since also Cs are incorporated in the platypus, other enzymatic activities may be involved. Furthermore, since one C-A mismatch remains in the edited form of the platypus tRNA transcribed from the upstream gene [24] and an A-A mismatch is created in the land snail tRNA ${ }^{\text {Tyr }}$ [23], the editing process seems not to be template directed from the 5 '-part of the tRNA acceptor stem, at least not in a simple Watson-Crick fashion.

\section{Editing of anticodons and import of tRNAs}

Overlapping tRNA genes are not the only case where mutated mitochondrial tRNAs are 'salvaged' by post-transcriptional mechanisms. In marsupial mitochondria, several tRNA genes encode transcripts that are not fully functional in their primary form. In particular, the sole aspartyl-tRNA gene encodes a gene product with the glycine anticodon GCC which is post-transcriptionally edited to the canonical aspartate anticodon GUC [27,28]. It has been shown that the non-edited version of this tRNA functions as a tRNA for glycine [29], that is probably needed to take over the function of the tRNA ${ }^{\mathrm{Gly}}$ (UCC) which may be only partially functional due to a substitution in the anticodon loop [30]. Thus, in the case of the genes for the tRNAs for aspartate and glycine, the functional consequences of at least two mutations in their genes are 'corrected' by an RNA editing mechanism affecting the anticodon of the aspartate tRNA. It thus seems that the maintenance of the (presumably) nuclearly encoded editing machinery is easier to achieve than the elimination of the substitutions in the two tRNA genes. Interestingly, also the editing of tRNA acceptor stems seems to be rather 'cheap' to maintain since a tRNA encoded by a gene in a land snail that does not overlap with other genes, but does so in closely related species, is still corrected by RNA editing [23]. The 'repair' of the tRNA in the former species has thus been maintained, although the gene has been translocated to a genomic location where the need for editing could be eliminated by backmutations.

The gene for the tRNA for lysine in the marsupial mitochondrial genome is yet another example of a heavily mutated gene. It exhibits an unusual secondary structure [16] and lacks the expected anticodon in several marsupial species. In this case, import of a nuclear encoded tRNA ${ }^{\text {Lys }}$ rather than RNA editing, may be the 'salvage' mechanism that ensures mitochondrial function (M.D., unpublished observation).

\section{Reversing the ratchet}

From the above it is clear that mitochondrial tRNA genes may be prone to deleterious mutations and that once such mutations have become fixed in the mitochondrial gene pool of a germline, they will not easily become eliminated again due to the absence of recombination. It furthermore seems that in many cases 'salvage mechanisms', particularly RNA editing systems, have evolved to compensate for mitochondrial mutations. How do the mechanisms that 'salvage' transcripts carrying deleterious mutations become fixed in mitochondria?

Since many copies of the mitochondrial genome are present within an animal cell, the effect of a mutation will initially be 
buffered by the presence of unmutated genomes. Studies from human diseases caused by mutations in mitochondrial tRNA genes show that as little as $10 \%$ of unmutated genomes is sufficient to ensure mitochondrial function [9]. However, since there is no way of eliminating a mutation by recombination, Müller's ratchet will apply. Thus, a mutation will linger on until the random process of genetic drift causes it either to be lost or to take over the entire population of mitochondrial genomes in a cell. If the mutation is serious enough, the cell would die were there no way to avoid the deleterious consequences of the mutation. Here, RNA editing provides a way out since it allows mitochondria to 'correct' potentially deleterious mutations. It is probable that the enzymatic mechanisms that change the transcripts are selected during the heteroplasmic period when mutations are present in only a fraction of the intracellular mtDNA pool and thus exert their effects only partially. During that period, enzyme(s) involved for example in cytidine deamination may have become recruited for the editing of the anticodon of the tRNA ${ }^{\text {Asp }}$ in marsupials [30].

Since a large part of the mutations that affect mitochondria are deletions [13], it may not be surprising that the most frequent type of editing found in animal mitochondria involves tRNAs whose genes overlap. Such overlaps would result from deletions that fall at boundaries between genes. In the cases where the upstream gene encodes a tRNA that can function with an $\mathrm{A}$ (or $\mathrm{C}$ ) residue as its discriminator base, an RNA editing activity inserting $A$ and $C$ residues at 3 '-ends of tRNAs may have become selected to 'patch up' the resultant transcripts. The phylogenetic tree in Fig. 3 shows where such overlaps may have been created (and eliminated by nucleotide insertions).

In conclusion, the RNA editing of $3^{\prime}$-ends of tRNA acceptor stems may have evolved as a result of the need to escape the effects of Müller's ratchet. Other such mechanisms are the editing of the anticodon of the tRNA ${ }^{\text {Asp }}$ in marsupials and the import of tRNAs from the cytoplasms in some groups of animals. Once a better understanding of the extent of genetic recombination and segregation in other organisms has been achieved, it is possible that also the editing of the 5'-parts of tRNA acceptor stems in Acanthamoeba [31] and some fungi [32], as well as the editing of mRNAs in trypanosomes, slimemoldes and plants may be found to be related to the workings of Müller's ratchet, even if other mechanisms may be more likely in some cases such as trypanosomal editing [33]. Additional organellar 'salvage pathways', which 'correct' the effects of genomic mutations are likely to be discovered in the future.

Acknowledgements: We are indebted to Hans Zischler, Markus Peintinger and Wilfried Gabriel for fruitful discussions, to the Alexandervon-Humboldt Stiftung for a fellowship to S.-I.Y. and to the Deutsche Forschungsgemeinschaft for financial support.

\section{References}

[1] Fischer-Lindahl, K., Trends Genet 1 (1985) 135-139.

[2] Gyllensten, U., Wharton, D., Josefsson, A., Wilson, A.C., Nature 352 (1991) 255-257.

[3] Kaneda, H., Hayashi, J., Takahama, S., Taya, C., Fischer-Lindahl, K., Yonekawa, H., Proc Natl Acad Sci USA 92 (1995) 4542-4546.

[4] Zouros, E., Oberhauser Ball, A., Saavedra, C., Freeman, K.R., Nature 368 (1994) 818.

[5] Zouros, E., Oberhauser Ball, A., Saavedra, C., Freeman, K.R., Proc Natl Acad Sci USA 91 (1994) 7463-7467.

[6] Stewart, D.T., Saavedra, C., Stanwood, R.R., Oberhauser Ball, A., Zouros, E., Mol Biol Evol 12 (1995) 735-747.

[7] Judson, O.P., Normark, B.B., Trends Ecol Evol 11 (1996) 41-45.

[8] Thyagarajan, B., Padua, R.A., Campbell, C., J Biol Chem 271 (1996) 27536-27543.

[9] Yoneda, M., Miyatake, T., Attardi, G., Mol Cell Biol 14 (1994) $2699-2712$.

[10] Müller, H.J., Mutat Res 1 (1964) 2-9.

[11] Felsenstein, J., Genetics 52 (1974) 737-756.

[12] Jenuth, J.P., Peterson, A.C., Fu, K., Shoubridge, E.A., Nature Genet 14 (1996) 146-151.

[13] Lynch, M., Mol Biol Evol 13 (1996) 209-220.

[14] Wolstenhome, D.R., Int Rev Cyt 141 (1992) 173-216.

[15] Kumazawa, Y., Nishida, M., J Mol Evol 37 (1993) 380-398.

[16] Janke, A., Feldmaier-Fuchs, G., Thomas, W.K., von Haeseler, A., Pääbo, S., Genetics 137 (1994) 243-256.

[17] Macey, J.R., Larson, A., Ananjeva, N.B., Papenfuss, T.J., Mol Biol Evol 14 (1997) 30-39.

[18] Kumazawa, Y., Yokogawa, T., Hasegawa, E., Miura, K.-I., Watanabe, K., J Biol Chem 264 (1989) 13005-13009.

[19] Kumazawa, Y., Schwartzbach, C.J., Liao, H.-X., Mizumoto, K., Kaziro, Y., Miura, K., Watanabe, K., Spremulli, L.L., Biochim Biophys Acta 1090 (1991) 167-172.

[20] Anderson, S., Bankier, A.T., Barrell, B.G., de Brujin, M.H.L., Coulson, A.R., Drouin, J., Eperon, I.C., Nierlich, D.P., Roe, B.A., Sanger, F., Schreier, P.H., Smith, A.J.H., Staden, R., Young, I.G., Nature 290 (1981) 457-465.

[21] Hatzoglou, E., Rodakis, G.C., Lecanidou, R., Genetics 140 (1995) 1353-1366.

[22] Ojala, D., Montoya, J., Attardi, G., Nature 290 (1981) 470-474.

[23] Yokobori, S., Pääbo, S., Proc Natl Acad Sci USA 92 (1995) $10432-10435$.

[24] Yokobori, S., Pääbo, S., Nature 377 (1995) 490.

[25] Tomita, K., Ueda, T., Watanabe, K., Nucl Acids Res 24 (1996) 4987-4991.

[26] Yokobori, S., Pääbo, S., J Mol Biol 265 (1997) 95-99.

[27] Janke, A., Pääbo, S., Nucl Acids Res 21 (1993) 1523-1525.

[28] Mörl, M., Dörner, M., Pääbo, S., Nucl Acids Res 23 (1995) $3380-3384$.

[29] Börner, G.V., Mörl, M., Janke, A., Pääbo, S., EMBO J 15 (1996) $5949-5957$.

[30] Börner, G.V., Pääbo, S., Nature 383 (1996) 225.

[31] Lonergan, K.M., Gray, M.W., Science 259 (1993) 812-815.

[32] Laforest, M.-J., Roewer, I., Lang, B.F., Nucl Acids Res 25 (1997) $626 \quad 632$.

[33] Cavalier-Smith, T., Trends Genet 13 (1997) 6-9.

[34] Gabriel, W., Lynch, M., Bürger, R., Evolution 47 (1993) 1744 1757. 